\title{
A Brief Study on Bhatia's Research of $L^{1}$-Convergence
}

\author{
바티의 $L^{1}$-수렴성 연구에 관한 소고
}

LEE Jung Oh 이정오

The $L^{1}$-convergence of Fourier series problems through additional assumptions for Fourier coefficients were presented by W. H. Young in 1913. We say that they are the classical results. Using modified trigonometric series is the convenience method to study the $L^{1}$-convergence of Fourier series problems. they are called the neoclassical results. This study concerns with the $L^{1}$-convergence of Fourier series. We introduce the classical and neoclassical results of $L^{1}$-convergence sequentially. In particular, we investigate $L^{1}$-convergence results focused on the results of Bhatia's studies. In conclusion, we present the research minor lineage of Bhatia's studies and compare the classes of $L^{1}$-convergence mutually.

Keywords: $L^{1}$-convergence, Fourier coefficients, trigonometric series; $L^{1}$-수렴성, 푸 리에 계수, 삼각급수.

MSC: 42A20, 42A32

\section{1 서론}

파키스탄 국경지역인 인도 북부 편자브(Punjab) 주 행정수도 파티알라(Patiala)시에 인도 최고 순위 공학대학 중 하나인 타파(Thapar) 대학교 $\left\langle\right.$ Figure 1 $>^{1)}$ 가 있다. 이곳 컴퓨터 수학 응용대학은 최근 10 년간 $L^{1}$-수렴성에 관한 연구활동이 가장 활발하게 진행되고 있는 곳 중 하나이다.

일반적으로 $L^{1}$ 거리공간에서 임의의 삼각급수의 함수가 $f \in L^{1}(T)$ 에 수렴하면 함수 $f$ 가 푸리에 급수라는 사실이 알려져 있다. 그러나 이 사실의 역은 성립하지 않는다. ${ }^{2)}$ 즉, 함수 $f$ 의 푸리에 급수가 $L^{1}$ 거리공간에서 수렴성을 항상 보장받지 못한다. 따라서 이 문제를 극복하기 위한 $L^{1}$-수렴성에 관한 연구를 많은 수학자들이 진행해 오고 있다.

LeE Jung Oh: Dept. of Math., Chosun Univ. E-mail: jolee@chosun.ac.kr Receiveded on Dec. 17. 2013, revised on Jan. 29. 2014, accepted on Feb. 5. 2014.

1) 사진출처 :http://www.thapar.edu

2) Riesz N. K. BArY, A treatise on trigonometric series, Vol I and Vol II, Pergamon Press, London, 1964, Vol. II, Ch VIII article 22. 


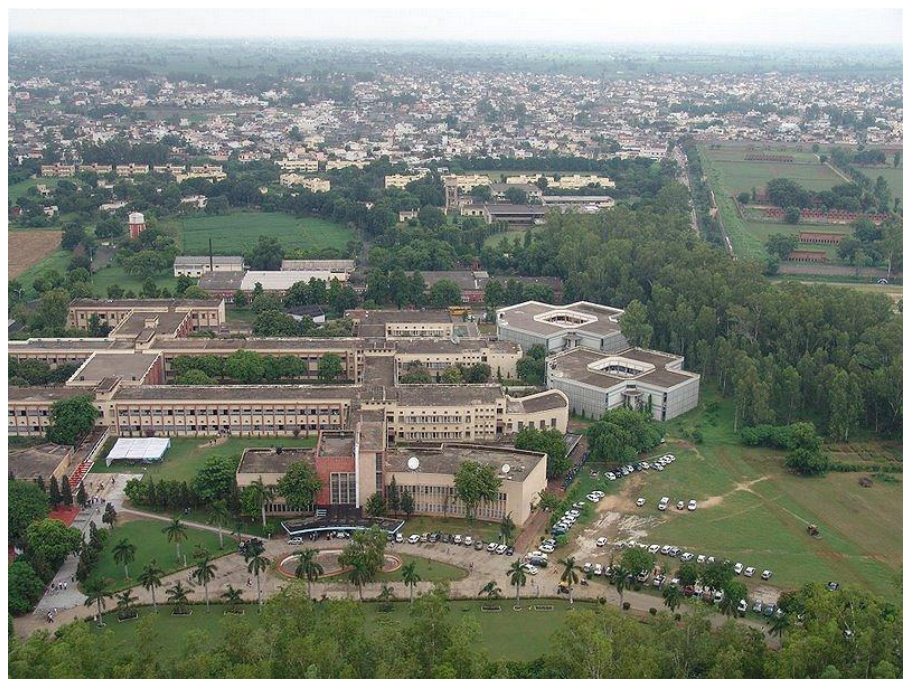

Figure 1. 타파(Thaper) 대학교 전경

본 논문의 2장에서 1913년 영 (W. H. Young)이 제시한 푸리에 급수의 $L^{1}$-수렴성 문제를 시작으로 1978년 포민과 테라코브스키의 연구까지 연구방향을 간략하게 살펴보고 3장에서는 스타노제빅 (Caslav V. Stanojevic)을 시작으로 활발하게 진행되어온 신 고전적인 결과들 중 바티 (S. S. Bhatia) 의 연구를 중심으로 논한다. 마지막으로 4장에서는 바티 (S. S. Bhatia)의 연구 소계보를 작성하고 $L^{1}$-수렴 푸리에 계수족들의 상호 관계성을 고찰한다.

2 고전적인 푸리에 급수의 $L^{1}$-수렴성에 관한 연구결과

$L^{1}$-수렴에 관한 문제의 핵심은 바나흐 공간인 $L^{1}$-공간에서는 적분 가능한 복소함수가 일반적 으로 노름에 대하여 수렴성을 보장하지 못하는 사실을 극복하기 위해 푸리에 계수들에 대한 부가적인 가정을 통해 다음 두 동치 조건식 (2)와 (3)을 만족하는 푸리에 계수의 성질들을 규명하는 연구로 진행되고 있다.

주어진 $T=\{t \in R \mid-\pi \leq t \leq \pi\}$ 상에서 르베그 적분가능한 모든 복소함수의 $L^{1}$-공간을 $L^{1}(T)$ 라 하고 $f \in L^{1}(T)$ 인 함수 $f$ 에 대한 노름(norm)은

$$
\|f\|_{L^{1}(T)}=\frac{1}{2 \pi} \int_{-\pi}^{\pi}|f(t)| d t
$$

로 정의되고 함수 $f$ 의 푸리에 급수 $S[f]$ 는

$$
S[f]=\sum_{|n| \leq \infty} \hat{f}(n) e^{i n t} \quad(n \in Z),
$$

이라한다. 여기서 $f$ 의 푸리에 계수는

$$
\hat{f}(n)=\int_{T^{1}} f(t) e^{-i n t} d t, \quad|n|<\infty
$$


이다. 또한 푸리에 급수 $S[f]$ 의 $n$-번째 부분합은

$$
S_{n}(f)=\sum_{|k| \leq n} \hat{f}(k) e^{i k t}, \quad n=0,1,2, \cdots .
$$

이다. 한편 푸리에 급수의 수렴문제는 간편성 때문에 푸리에 코사인 급수만을 가지고 수렴성을 보이는데 푸리에 코사인 급수

$$
f(t)=\frac{a_{0}}{2}+\sum_{n=1}^{\infty} a_{n} \cos n t
$$

의 $f \in L^{1}(0, \pi)$ 수렴성 문제는 푸리에 계수 $a_{n}$ 즉

$$
a_{n}=\frac{1}{\pi} \int_{-\pi}^{\pi} f(t) \cos n t d t
$$

의 성질을 찾는 일로 귀결되는데

$$
\left\|S_{n}(f)-f\right\|_{L^{1}}=o(1), \quad n \rightarrow \infty
$$

(2) 와 필요충분조건인 다음 (3)식

$$
a_{n} \log n=o(1), \quad n \rightarrow \infty
$$

을 만족하는 푸리에 계수를 이용하여 푸리에 급수의 $L^{1}$-수렴성을 규명하는 연구가 진행되고 있다.

1913년 영 (W. H. Young) 이 푸리에 계수가 볼록인

$$
\Delta^{2} a_{n} \geq 0
$$

인 경우 동치조건 (2)와 (3)을 만족한다는 사실을 밝혔는데 이 볼록 계수를 좀 더 일반화하여 푸리에 계수가 준 볼록

$$
\sum_{n=1}^{\infty}(n+1)\left|\Delta^{2} a_{n}\right|<\infty
$$

인 경우를 1923 년 콜모고로프(A. N. Kolmogorov) $)^{3)}$ 가 밝혔다. 다시 1939년 시던(S. Sidon) ${ }^{4)}$ 은 1939년 유계변동

$$
\sum_{n=1}^{\infty}\left|\Delta^{m} a_{n}\right|<\infty, \quad m \geq 1
$$

인 영 (null) 계수수열 $S$ 족(시던족)

$$
\sum_{n=1}^{\infty}\left|\Delta a_{n}\right|<\infty
$$

을 정의하여 콜모고로프의 모든 준 볼록 계수가 $S$ 족임을 보임으로서 콜모고로프의 준 볼록 계수를 더 확장하였다. 결론적으로 $S$ 족은 유계변동 족의 부분집합이 된다[18].

3) A. N. Kolmogorov, Sur l'ordre de grandeur des coefficients dela serie de Fourier-Lebesgue, Bull. Internat. Acad. Polon. Sci. Lett. CI. Sci. Math. Nat. Ser. A Sci. Math. (1923), 83-86.

4) S. Sidon, Hinreichende Bedingungen fur den Fourier charakter einer trigonometrischen Reihe, J. London Math. Soc. 14(1939), 158-160. 
한편, 일본 나가노 현에 있는 신슈(Shinshu) 국립대학 카노(T. Kano) ${ }^{5)}$ 교수는 1968년 “어떤 삼각급수의 계수” 라는 연구 결과를 발표하였는데 이를 재해석하면

"만약 임의의 영 (null) 수열 $\left\{a_{k}\right\}$ 이

$$
\sum_{k=1}^{\infty} k^{2}\left|\Delta^{2}\left(\frac{a_{k}}{k}\right)\right|<\infty
$$

를 만족하면 $\left\{a_{k}\right\}$ 은 $T$ 족에 속한다.” 라고 분류하였는데 그는 $L^{1}$-수렴성 문제를 더 확장하고 일반화하는데 기여한다.

이어 1973년 테라코브스키 (S. A. Telyakovskii) ${ }^{6}$ )는 $S$ 족 조건을 더욱 간단 명료한 동치형 태로 표현하고 $L^{1}$-수렴성 문제에 대하여 $S$ 족 조건을 이용하여 폭넓게 규명하는데 기여하게 된다.

1975년 포민과 테라코브스킥)는 준 단조계수

$$
\frac{a_{n}}{n^{\alpha}} \downarrow, \quad n \rightarrow \infty, \quad \alpha \geq 0
$$

를 가진 푸리에 급수에 관해 소개하고 포민은 3년뒤 이 연구결과를 더욱 일반화하여 1978 년8)또 다른 연구결과를 발표하게 된다[19]. 결국, 이들의 고전적인 연구결과들은 푸리급수의 $L^{1}$-수렴성 문제를 해결하는데 푸리에 계수로 이루어진 수열에 대한 부가적인 가정을 통해 수렴성을 보이고 있다.

\section{3 신 고전적인 푸리에 급수의 $L^{1}$-수렴성에 관한 연구결과}

$L^{1}$-수렴성 문제를 논하는데 있어서 기존 고전적인 방법과 다른 변형된 삼각급수를 소개하여 수렴성을 용이하게 접근하고 있는데 이러한 신 고전적인 연구를 제시한 사람들은 주로 스 타노제빅 (C. V. Stanojevic), 람(B. Ram), 싱과 샤르마(N. Singh and K. M. Sharma), 첸 (C. P. Chen), 토머브스키 (Z. Tomovski), 카어 (K. Kaur) 그리고 바티 (S. S. Bhatia) 등을 들 수 있다. 이 부분에 많은 연구를 진행했던 스타노제빅[20] 의 연구 이후 2000년 들어 인도의 바티와 카어 그리고 람은 $L^{1}$-수렴성 연구를 매우 활발하게 진행한 연구자들로 여겨진다. 인도 최고 공학대학 중 하나인 타파 (Thapar) 대학교 컴퓨터 수학 응용대학의 바티 (S. S. Bhatia) 교수는 1993년부터 2012년까지 관련논문만 30편 이상을 발표하고 있다. 특히, 이절에서는 바티의 연구를 중심으로 그의 연구 결과들을 재해석 고찰한다.

바티 (Satvinder singh Bhatia) $<$ Figure 2>9)는 인도 로타크(Rohtak)시에 있는 마하리쉬

5) T. Kano, Coefficients of some trigonometric series, J. Fac. Sci. Shinshu Univ. 3(1968), 153-162.

6) S. A. TelyakovskiI, Concerning a sufficient condition of Sidon for the integrability of trigonometric series, Math, Notes 14 (1973), 742-748.

7) G. A. Fomin and S. A. TelyakovskiI, On the convergence in L metric of Fourier series with quasimonotone coefficients, Trudy Math. Inst. Steklov. 134 (1975), 310-313.

8) G. A. Fomin, A class of trigonometric series, Math. Notes 23 (1978), 117-123.

9) 사진출처 : http://www.thapar.edu/faculty 


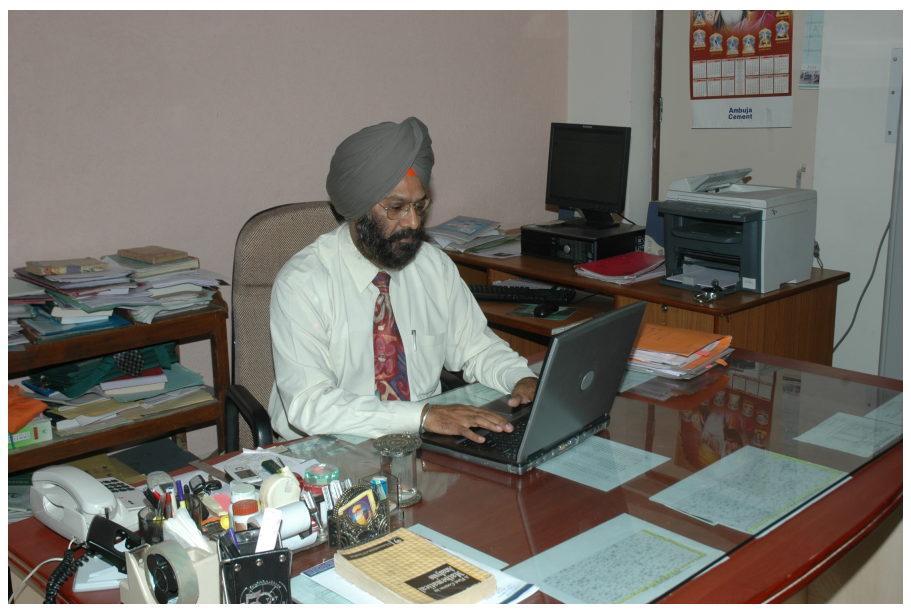

Figure 2. 바티 (Satvinder singh Bhatia)

(Maharishi) 대학에서 스승 람(B. Ram)으로부터 1993년 "특별한 계수를 가진 삼각급수의 $L^{1}$-수렴성과 적분가능성”[1] 논문으로 학위를 받고 푸리에 급수 $L^{1}$-수렴성 연구자들에게 주목을 받게 된다. 그 해 바티 (S. S. Bhatia)는 스승 람(B. Ram) 과 공동연구를 통해 1993년 “어떤 변형된 삼각계 총합의 $L^{1}$-수렴성에 관하여”[2]라는 연구를 내놓는다. 만약 임의의 영 (null) 수열 $\left\{a_{k}\right\}$ 이

$$
\sum_{k=1}^{\infty} k^{r+2}\left|\Delta^{2}\left(\frac{a_{k}}{k}\right)\right|<\infty
$$

임을 만족하면 $\left\{a_{k}\right\}$ 은 $T_{r}$ 족에 속한다고 분류하였다. 이 결과를 재해석하면 $r=0$ 이면 이들이 연구한 결과는 $T_{r}$ 족은 카노의 결과와 같은 $T$ 족이 된다. 1995 년 그들은 2 년간의 공동 연구를 통해 $T$ 족을 이용한 $T_{r}$ 족의 연구를 통해 복소 영 (null) 수열을 소개하고 $T^{*}$ 족을 연구 “변형된 복소 삼각계 총합의 $L^{1}$-수렴성에 관하여”[3]을 발표한다. 이 논문은 만약 임의의 복소 영 (null) 수열 $\left\{a_{k}\right\}$ 이 다음 (4)와 (5) 두 조건을 만족하면

$$
\begin{aligned}
& \sum_{k=1}^{\infty}\left|\Delta\left(\frac{a_{-k}-a_{k}}{k}\right)\right| k \log k<\infty, \\
& \sum_{k=1}^{\infty} k^{2}\left|\Delta^{2}\left(\frac{a_{k}}{k}\right)\right|<\infty .
\end{aligned}
$$

$\left\{a_{k}\right\}$ 은 $T^{*}$ 족에 속하는 분류임을 보인것이다. 그리고 다음해인 1996년 그들은 "모리츠(F. Moricz) 정리의 확장” [4] 연구를 통해 변형된 삼각계 총합을 이용 $r$ 번 미분된 복소 삼각급수 의 적분가능성과 $r$ 도함수를 가진 푸리에 급수의 $L^{1}$-수렴성에 관한 새로운 필요충분조건을 제시한다. 이 결과는 모리츠의 정리를 더 확장한 논문으로 만약 어떤 영 (null) 수열 $\left\{a_{k}\right\}$ 이

$$
\sum_{k=1}^{\infty}\left|\Delta\left(a_{k}-a_{-k}\right)\right| k^{r} \log k<\infty, \quad r=0,1,2, \cdots
$$


을 만족하면 $\left\{a_{k}\right\}$ 은 $W_{r}$ 족에 속한다고 분류하고 수열 $\left\{a_{k}\right\} \in W_{r}$ 이고

$$
\sum_{m=1}^{\infty} 2^{m(1 / q+r)}\left(\sum_{k \in I_{m}}^{\infty}\left|\Delta a_{k}\right|^{p}\right)^{\frac{1}{p}}<\infty, \text { 단, } 1<p<2,0 \leq r
$$

을 만족하면 다음 두 조건 (6) 과 (7)이 서로 필요충분조건이 성립함을 보인것이다.

$$
\begin{aligned}
& \left\|S_{n}^{(r)}(f)-f(r)\right\|_{L^{1}}=o(1), \quad n \rightarrow \infty, \\
& |n|^{r} \hat{f}(n) \log |n|=o(1), \quad|n| \rightarrow \infty .
\end{aligned}
$$

이때, $S_{n}^{(r)}(f)$ 은 부분합 $S_{n}(f)$ 의 $r$ 번째 도함수이다. 다음해 1997에도 그들은 다시 모리츠의 연구를 확장한 “이중 코사인 급수의 가중 적분”[5]을 발표한다. 그리고 바티 (S. S. Bhatia)는 마하리쉬 (Maharishi) 대학의 람과 그리고 그의 학위과정 중인 제자 카어(K. Kaur)와 공동 연구를 수행하여 1975년 리스-스타노제빅 (Rees-Stanojevic) ${ }^{10)}$ 에 의해 소개된 "변형된 코사인 총합의 $L^{1}$-수렴성과 적분가능성” 에 대해 적분차수의 세자로(Cesaro) 평균을 이용 일반화된 준 볼록 영 (null) 계수족 연구를 통해 "일반화된 준 볼록 계수를 가진 리스-스타노제빅 총합의 $L^{1}$-수렴성과 적분가능성”[6]을 2002년에 발표한다. 즉,

“만약 $\left\{a_{n}\right\}$ 어떤 일반화된 준 볼록 영 (null) 수열이면 $g_{n}(x)$ 은 $g(x)$ 에 $L^{1}$ 거리공간에서 수렴한다는 것과 필요충분조건이

$$
\lim _{n \rightarrow \infty} \Delta a_{n} \log n=o(1), n \rightarrow \infty
$$

이 된다.”를 밝힌 것이다. 여기서

$$
g(x)=\frac{a_{0}}{2}+\sum_{n=1}^{\infty} a_{n} \cos n x
$$

이고

$$
g_{n}(x)=\frac{1}{2} \sum_{k=0}^{n} \Delta a_{k}+\sum_{k=1}^{n} \sum_{j=k}^{n}\left(\Delta a_{j}\right) \cos k x
$$

이며 $g_{n}(x)$ 와 $a_{n}$ 의 관계식은

$$
g_{n}(x)=S_{n}(x)-a_{n+1} D_{n}(x)
$$

이다. 한편, 카어는 이 연구를 시작으로 2 편의 논문을 더 발표하고 스승 바티로부터 2003년 박사학위를 받게된다. 같은 해인 2002년에도 람과 같은 대학 동료교수인 후다(N. Hooda)를 포함한 3명이 공동연구를 진행하여 “어떤 변형된 코사인 총합의 $L^{1}$-수렴성에 관하여”[7]를 발표하는데 이는 임의의 준 볼록 영 (null) 수열이 $S$ 족 속한다는 성질을 이용한 것으로 이를 재해석하면 만약 코사인 급수 (1)의 계수수열 이 $S$ 족에 속하면 $L^{1}$-수렴성에 대한 필요충분조 건이

$$
a_{k} \log k=o(1), \quad k \rightarrow \infty
$$

10) J. W. Garrett and Č. V. Stanojević, On integrability and $L^{1}$-convergence of certain cosine sums, Notices Amer. Math. Soc. 22 (1975), (A-166). 
임을 보인 것이다.

2003년에는 카어(K. Kaur)와 공동연구를 통해 2편의 논문을 발표한다. 먼저 브레이(W. O. Bray)의 결과를 복소 이중 푸리에 급수로 확장하여 “복소 이중 푸리에 급수의 $L^{1}$-수렴성” [8]을 소개한다. 바티(S. S. Bhatia)는 람(B. Ram)과 카어(K. Kaur)와 함께 공동연구를 통해 람과 쿠마리(S. Kumari) 가 1989년 소개했던 “특정 삼각계의 $L^{1}$-수렴성에 관하여” ${ }^{11)}$ 의 결과에 이용 새로운 $K^{*}$ 족 계수를 갖는 푸리에 급수의 $L^{1}$-수렴성에 대한 필요충분조건을 얻게되는데 즉 “변형된 복소 삼각계 총합의 $L^{1}$-수렴성”[9]의 결과를 발표한다.

이 논문은 만약 $\left\{a_{n}\right\}$ 이 $K^{*}$ 족에 속하면 (2)와 (3) 두 조건은 서로 필요충분조건임을 보인 것이다.

2004 년에도 람과 카어와 더불어 2 편의 결과를 발표한다. 먼저, 그들은 새로 변형된 사인 총합을 소개하고 새로운 $K$ 족에 속한 변형된 사인 총합이 $L^{1}$-수렴성임을 보인다. 그들의 연구 결과는 “어떤 삼각계 총합의 $L^{1}$-수렴성에 관하여”[10]이다. 그리고 세 사람은 연이어 “고차 유계 변동 계수를 가진 이중 삼각급수”[11] 결과를 발표한다.

한편, 바티교수는 제자 자틴더디프 카어(Jatinderdeep Kaur) 의 학위과정부터 줄곧 연구를 같이하여 람이 발표한 “거리공간 $\mathrm{L}$ 에서 특정코사인 총합의 수렴성” ${ }^{12)}$ 을 확장하고 $r$-번째 도함수를 가진 코사인 급수의 $L^{1}$-수렴성을 연구하여 2007년 "특정 코사인총합의 $L^{1}$-수렴성과 적분가능성”[12]을 소개한다. 만약 (1)이 $S_{r}$ 족에 속하면,

$$
\left\|f-g_{n}\right\|_{L^{1}}=o(1), \quad n \rightarrow \infty .
$$

성립함을 보인것이다. 여기서 $S_{r}, \quad r=1,2,3, \cdots$ 는 새로운 시던 족(Sidon class)을 의미 하며

$$
A_{k} \downarrow 0, \quad k \rightarrow \infty, \quad \sum_{k=0}^{\infty} k^{r} A_{k}<\infty
$$

이고

$$
\left|\Delta a_{k}\right| \leq A_{k}
$$

을 만족하는 임의의 수열 $\left\{A_{k}\right\}$ 이 존재할때 영 (null) 수열 $\left\{a_{k}\right\}$ 이 $S_{r}$ 에 속한다고 말한다. 결국 $S_{r+1} \subset S_{r}$ 임을 알수 있다. 다시 2008년 카어(J. Kaur)와 “거리공간 $L$ 에서 새롭게 변형된 삼각계 총합의 수렴성”[13]에서 새롭게 변형된 코사인과 사인총합을 소개하였는데 그 형태는

$$
\frac{a_{0}}{2}+\sum_{k=1}^{n} \sum_{j=k}^{n} \Delta\left(a_{j} \cos j x\right)
$$

11) B. Ram and S. Kumari, On $L^{1}$-convergence of certain trigonometric sums, Indian J. Pure Appl. Math. 20(9) (1989), 908-914.

12) B. RAm, Convergence of certain cosine sums in the metric space L, Proc, Amer. Math. Soc. 66(2) (1977), 258-260. 
와

$$
\sum_{k=1}^{n} \sum_{j=k}^{n} \Delta\left(a_{j} \sin j x\right)
$$

인데 $L^{1}-$ 수렴성과 적분가능성을 연구한 것이다. 주정리 중 하나를 살펴보면

“만약 코사인 급수 식 (1)의 계수수열 $\left\{a_{n}\right\}$ 이 $S$ 족에 속하면 식 (1)의 $L^{1}$ 수렴성에 대한 필요충분조건으로 (8)을 만족함” 을 보인 것이다.

카어 (Jatinderdeep Kaur) 에게 박사학위를 수여한 해인 2009년에는 가렛(J. W. Garrett), 리스(C. S. Rees) 그리고 스타노제빅(C. V. Stanojevic) 이 발표한 1980년의 “유계변동 계수를 가진 푸리에 급수의 $L^{1}$-수렴성” ${ }^{13)}$ 을 일차원에서 이차원으로 확장한 “가렛, 리스와 스타노 제빅의 정리를 1 차원에서 2차원으로 확장”[14] 을 소개한다. 또한 2008년에 연구한 결과를 복소형태로 확장하여 새롭게 변형된 코사인과 사인총합을 연구하여 2010년 카어(J. Kaur)와 “거리공간 L에서 변형된 복소 삼각계 총합의 수렴성”[15]이라는 제목으로 발표한다. 재해석 하면, 복소수열 $\left\{c_{k}\right\}$ 이 $\left\{c_{k}\right\} \in J^{*}$ 이면, $|t| \in(0, \pi]$ 에 대하여

$$
\lim _{n \rightarrow \infty} g_{n}(C, t)=f(t)
$$

이 존재하고

$$
f(t) \in L_{1}(0, \pi]
$$

에 대하여

$$
\left\|f-g_{n}\right\|_{L^{1}}=o(1), \quad n \rightarrow \infty
$$

와 필요충분조건이

$$
\left\|S_{n}(C, t)-f(t)\right\|_{L^{1}}=o(1), \quad n \rightarrow \infty
$$

임을 발표한것이다. 여기서 (9)와

$$
\left|\Delta\left(\frac{c_{k}-c_{-k}}{k}\right)\right| \leq \frac{A_{k}}{k}
$$

를 만족하는 임의의 $\left\{A_{k}\right\}$ 가 존재하면 영 (null) 수열 $\left\{c_{n}\right\}$ 이 $J^{*}$ 에 속한다고 정의한다. 또한 복소수 형태의 합인 $g_{n}(C, t)$ 는

$$
\begin{gathered}
g_{n}(C, t)=S_{n}(C, t)+\frac{i}{2 \sin t}\left[c_{n} e^{i(n+1) t}-c_{-n} e^{-i(n+1) t}+c_{n+1} e^{i n t}-c_{-(n+1)} e^{-i n t}\right. \\
\left.+\left(c_{n}-c_{n+2}\right) E_{n}(t)+\left(c_{-(n+2)}-c_{-n}\right) E_{-n}(t)\right]
\end{gathered}
$$

이고 $E_{n}(t)=\sum_{k=0}^{n} e^{i k t}$ 이다. 이듬해인 2011년 두 사람은 "이중 코사인 삼각급수의 $L^{1}$ 수렴성과 적분가능성”[16]에 대하여 연구하고 최근 2012년에는 “새로운 변형 코사인 합의 $L^{1}$ 수렴성인 어떤 족”[17] 에 대한 결과를 소개한 바 있다.

13) J. W. Garrett, C. S. Rees and C. V. Stanojevic, $L^{1}$-convergence of Fourier series with coefficients of bounded variation, Proc. Amer. Math. Soc. 80(3) (1980), 423-430. 


\section{4 결론 : 바티(S. S. Bhatia) 중심의 $L^{1}$ 수렴성 결과들과 연구 소계보}

부가적인 가정을 통해 푸리에 계수들의 특징을 분류하여 $L^{1}$-수렴성을 보이는 연구는 많이 연구 되어 오고있다. 3장에서 조사된 바티(S. S. Bhatia) 연구를 바탕으로 이 절에서는 결론으로서 $L^{1}$-수렴성 푸리에 계수족들 중 반볼록 계수수열을 분류하고 상호 포함관계를 재해석하고 바티의 $L^{1}$-수렴성 연구에 대한 소계보〈Figure $3>$ 를 작성한다.

만약 임의의 영 (null) 수열

$$
\left\{a_{k} \rightarrow 0, \quad k \rightarrow \infty\right\}
$$

이고

$$
\sum_{k=1}^{\infty} k\left|\Delta^{2} a_{k-1}+\Delta^{2} a_{k}\right|<\infty
$$

를 만족할 때 $\left\{a_{k}\right\}$ 을 반볼록 수열 (semi-convex : SC)이라 하고 간단히 $\left\{a_{k}\right\} \in\{S C\}$ 로 표시한다. 여기서

$$
\Delta^{2} a_{k}=a_{k}-2 a_{k+1}+a_{k+2}
$$

이고 다시 이 반볼록 수열 (SC) 인 $\left\{a_{k}\right\}$ 을 더 일반화하여 $r$ 차 반볼록 수열을 $\{S C\}^{r}$ 로 표현 할 수 있다. 즉 만약 임의의 영 (null) 수열이

$$
\left\{a_{k} \rightarrow 0, k \rightarrow \infty\right\}
$$

이고

임을 만족하면

$$
\sum_{k=1}^{\infty} k^{r+2}\left|\Delta^{2} a_{k-1}+\Delta^{2} a_{k}\right|<\infty
$$

$$
\left\{a_{k}\right\} \in\{S C\}^{r} \quad r=0,1,2, \cdots
$$

라고 한다. 결과적으로

$$
\{S C\} \subset\{S C\}^{r}
$$

이고 $r=0$ 이면 $\{S C\}=\{S C\}^{r}$ 이다.

한편, 1987년 발라와 람(R. Bala, and B. Ram) ${ }^{14)}$ 은 반볼록 영 (null) 계수를 가진 코사인 삼각급수가 다음 정리가 성립함을 보인다. 즉 만약 $\left\{a_{k}\right\} \in\{S C\}$ 이면 (1)에 대한 $L^{1}$ 거리공 간에서 수렴하는 필요충분조건이

$$
a_{k-1} \log k=o(1), \quad k \rightarrow \infty
$$

임을 보인다.

바티는 공동연구를 통해 2002년 일반화된 반볼록 계수인

$$
k^{\alpha}\left|\Delta^{\alpha+1} a_{k-1}+\Delta^{\alpha+1} a_{k}\right|<\infty
$$

14) R. BAlA, and B. RAM, Trigonometric series with semi-convex coefficients, Tamkang J. Math. 18(1) (1987), 75-84 
을 만족하고 계수수열이

$$
\left\{a_{k}\right\} \rightarrow 0, k \rightarrow \infty
$$

일때 $\left\{a_{k}\right\}$ 이 \{GSC; generalized semi-convex\}에 속함을 보이는데 이것은 일반화된 반 볼록 계수를 가진 리-스타노제빅 총합의 $L^{1}$-수렴성과 적분가능성에 관한 연구이다. 이어서 2004년 바티는 공동연구를 통해 준 볼록 계수를 가진 새로 변형된 사인 총합 $K_{n}(x)$ 의 $L^{1}$-수렴성을 연구한 정리이다. 다시말하면

$$
\left\{a_{k}\right\} \in\{S C\}
$$

이라 하면 $L^{1}$-노름 공간에서 $K_{n}(x)$ 가 $f(x)$ 에 수렴함을 보인것이다. 그리고 새로 변형된 사인 총합 $K_{n}(x)$ 은

$$
K_{n}(x)=\frac{1}{2 \sin x} \sum_{k=1}^{n} \sum_{j=k}^{n}\left(\Delta a_{j-1}-\Delta a_{j+1}\right) \sin k x
$$

이고 여기서

$$
\Delta a_{j}=a_{j}-a_{j+1}
$$

이다.

2010년 바티는 카어와 함께 2004년 연구한 새로 변형된 사인 총합 $K_{n}(x)$ 을 복소 형태로 소개하고 최근 이 두 사람은 2012년에는 계수 수열의 임의의 새로운 족에 대한 소개된 변형 코사인 삼각계 총합을 새롭게 소개함으로서 사인 삼각급수의 $L^{1}$-수렴성을 연구중에 있다. 
바티의 $L^{1}$-수렴성에 관한 연구 소계보

\begin{tabular}{|c|c|}
\hline 특별한 계수를 가진 삼각급수의 $L^{1}$-수렴성과 적분가능성 & 1993 \\
\hline 어떤 변형된 삼각계 총합의 $L^{1}$-수렴성에 관하여 & 1993 \\
\hline 변형된 복소 삼각계 총합의 $L^{1}$-수렴성에 관하여 & 1995 \\
\hline 모리츠(F. Moricz) 정리의 확장 & 1996 \\
\hline 이중 코사인 급수의 가중 적분 & 1997 \\
\hline $\begin{array}{l}\text { 일반화된 준 볼록 계수를 가진 리스-스타노제빅 총합의 } \\
L^{1} \text {-수렴성과 적분가능성 }\end{array}$ & 2002 \\
\hline 어떤 변형된 코사인 총합의 $L^{1}$-수렴성에 관하여 & 2002 \\
\hline 복소 이중 푸리에 급수의 $L^{1}$-수렴성 & 2003 \\
\hline 변형된 복소 삼각계 총합의 $L^{1}$-수렴성 & 2003 \\
\hline 변형된 사인 총합의 $L^{1}$-수렴성과 적분가능성 & 2004 \\
\hline 고차 유계 변동 계수를 가진 이중 삼각급수 & 2004 \\
\hline 특정 코사인총합의 $L^{1}$-수렴성과 적분가능성 & 2007 \\
\hline 거리공간 L에서 새롭게 변형된 삼각계 총합의 수렴성 & 2008 \\
\hline 가렛, 리스와 스타노제빅의 정리를 1 차원에서 2 차원으로 확장 & 2009 \\
\hline 거리공간 L에서 변형된 복소 삼각계 총합의 수렴성 & 2010 \\
\hline 이중 코사인 삼각급수의 $L^{1}$-수렴성과 적분가능성 & 2011 \\
\hline 새로운 변형 코사인 합의 $L^{1}-$ 수렴성 족 & 2012 \\
\hline
\end{tabular}

Figure 3. 바티의 $L^{1}$-수렴성에 관한 연구 소계보 


\section{참고 문헌}

1. S. S. Bhatia, Integrability and $L^{1}$-convergenece of trigonometric series with special coefficients, Ph. D Thesis, M. D. University, Rohtak, India, 1993.

2. S. S. BнAтіA and B. RAM, On $L^{1}$-convergence of certain modifed trigonometric sums, Indian J. Math. 35(2) (1993), 171-176.

3. S. S. Bhatia and B. RAM, On $L^{1}$-convergence of modifed complex trigonometric sums, Proc. Indian Acad. Sci. Math. Sci. 105(2) (1995), 193-199.

4. S. S. Bhatia and B. Ram, The extensions of the F. Moricz theorems, Proc. Amer. Math. Soc. 6(124) (1996), 1821-1829.

5. S. S. Bhatia and B. Ram, On weighted Integrability of double cosine series, J. of Mathematical Analysis and Applications 208 (1997), 510-519.

6. Kulwinder KAUR and S. S. BhATIA, Integrability and $L^{1}$-convergence of Rees-Stanojevic sums with generalized semiconvex coefficients, IJMMS 30:11 (2002), 645-650.

7. N. Hooda, B. Ram and S. S. BHATIA, On $L^{1}$-convergence of a modified cosine sum, Soochow J. of mathematics 28(3) (2002), 305-310.

8. K. Kaur, S. S. Bhatia and B. Ram, $L^{1}$-convergence of complex double Fourier series, Proc. Indian Acad. Sci. Math. Sci. 113(4) (2003), 355-363.

9. S. S. Bhatia, Kulwinder Kaur and B. Ram, $L^{1}$-convergence of modified complex triginometrc sums, Lobachevskii Journal of Mathematics 12 (2003), 3-10.

10. K. KaUR, S. S. Bhatia, and B. RAM, On $L^{1}$-convergence of certain trigonometric sums, Georgian Math. J. 11(1) (2004), 99-104.

11. K. Kaur, S. S. Bhatia, and B. Ram, Double trigonometric series with coefficients of bounded variation of higher order, Tamkang J. Math. 35(4) (2004), 267-280.

12. J. KaUR and S. S. Bhatia, Integrability and $L^{1}$-convergence of certain cosine sums, Kyungpook Mathematical Journal 47 (2007), 323-328.

13. J. KAUR and S. S. Bhatia, Convergence of new modified trigonometric sums in the metric space $L^{1}$, The Journal of Nonlinear Sciences and Applications 1(3) (2008), 179-188.

14. Jatinderdeep Kaur and S. S. Bhatia, The extension of the theorem of J. W. Garrett, C. S. Rees and C. V. Stanojevic from one dimension to two dimension, Int. Journal of Math. Analysis 3(26) (2009), 1251-1257.

15. J. KaUR, S. S. Bhatia, Convergence of modified complex trigonometric sums in the metric space L, Lobacherskii J. Math. 31(3) (2010), 290-294.

16. J. KAUR and S. S. BhATIA, Integrability and $L^{1}$-convergence of double cosine trigonometric series, Anal. Theory Appl. 27(1) (2011), 32-39.

17. J. KaUR, S. S. Bhatia, A class of $L^{1}$-convergence of new modified cosine sums, Southeast Asian Bull. Math. 36 (2012), 831-836.

18. LeE Jung Oh, The life of Fourier, The minor Lineage of his younger scholars and a theorem of Telyakovskii on $L^{1}$-convergence, The Korean Journal for History of Mathematics 22(1) (2009), 25-40.

19. LeE Jung Oh, Partial sum of Fourier series, the reinterpret of $L^{1}$-convergence results using Fourier coefficients and theirs minor lineage, The Korean Journal for History of Mathematics 
23(1) (2010), 53-66.

20. LeE Jung Oh, A brief study on Stanojevic's works on the $L^{1}$-convergence, The Korean Journal for History of Mathematics 26(2-3) (2013), 163-176. 